\title{
Factores asociados con la conversión de la colecistectomía laparoscópica a colecistectomía abierta
}

\section{Factors Associated with Conversion of Laparoscopic Colecistectomy to Open Colescytectomy}

\author{
Ledmar Vargas R., ${ }^{1}$ Milena Agudelo S., ${ }^{1}$ Roger Lizcano C., ${ }^{1}$ Melissa Martínez B., ${ }^{1}$ Leonardo Velandia B., ${ }^{1}$ Sergio Sánchez H., ${ }^{1}$ \\ Óscar Jiménez P., PhD, ${ }^{2}$ Marco Quintero, MD. ${ }^{3}$
}

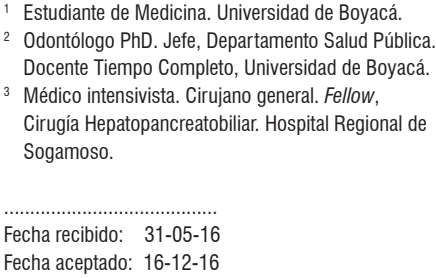

Fecha recibido: $\quad 31-05-16$

Fecha aceptado: $16-12-16$

\begin{abstract}
Resumen
Introducción: la colecistitis aguda es una inflamación de la pared vesicular. El tratamiento para esta patología es netamente quirúrgico, y la colecistectomía laparoscópica es el procedimiento de elección. Esta puede suffir conversión intraoperatoria debido a las complicaciones propias de la intervención, los factores del paciente 0 los asociados con el cirujano. Objetivo: el objetivo del estudio es establecer la frecuencia y la asociación de conversiones en colecistectomía laparoscópica, basándonos en los exámenes de laboratorio hematológicos y la ecografía abdominal, así como en los factores sociodemográficos. Materiales y métodos: se realizó un estudio descriptivo, de corte transversal, observacional y retrospectivo, durante el período comprendido entre el 1 de enero y el 3 de noviembre de 2015. Se revisaron historias clínicas con diagnóstico de patología vesicular benigna de pacientes sometidos a procedimiento quirúrgico vesicular. Resultados: al $35,5 \%$ de los pacientes se les realizó una colecistectomía laparoscópica $(\mathrm{CL})$, en la que fue necesario convertir a cirugía convencional al $42,8 \%$ de los pacientes. La mayoría de los pacientes intervenidos fueron mujeres $(72,8 \%)$, aunque la conversión predominó en el sexo masculino. El diagnóstico preoperatorio más frecuente fue la colelitiasis $(98,3 \%)$. La edad mayor de 50 años presentó un odds ratio de 0,55, mientras que la leucocitosis presentó un odds ratio de 0,40; ambas variables fueron estadísticamente significativas $(p \leq 0,05)$. Conclusiones: se determinó que una edad mayor de 50 años y/o un valor de leucocitos mayor de $10000 \mathrm{~mm}^{3}$ son factores de riesgo para que una colecistectomía laparoscópica falle; además, deben tenerse en cuenta los factores propios del cirujano.
\end{abstract}

Palabras clave

Colecistectomía, colecistectomía laparoscópica, leucocitos, conversión a cirugía abierta, complicaciones.

\begin{abstract}
Acute cholecystitis is an inflammation of the vesicular wall whose treatment is purely surgical: a laparoscopic cholecystectomy is the procedure of choice. This can be converted intraoperatively due to complications of the intervention, patient factors or factors associated with the surgeon. The aim of this study was to establish the frequency and the association of conversions of laparoscopic cholecystectomies based on hematological laboratory tests and abdominal ultrasound as well as sociodemographic factors. Materials and Methods: This is a descriptive, cross-sectional, observational and retrospective study that was carried out from January 1 to November 3, 2015. Clinical histories of patients who had been with diagnosed with benign vesicular pathologies who underwent vesicular surgical procedures were reviewed. Results: Of the cases reviewed, $35.5 \%$ patients underwent laparoscopic cholecystectomies, and of these cases $42.8 \%$ of the procedures were converted to conventional surgery. The majority of the patients were women $(72.8 \%)$, but conversions were most frequent among men. The most frequent preoperative diagnosis was cholelithiasis $(98.3 \%)$. Age greater than 50 years presented an odds ratio of 0.55 , while leukocytosis had an odds ratio of 0.40 , both variables were statistically significant $(P=<0.05)$. Conclusions: It was determined that ages over 50 years and/or a leukocyte count over $10,000 \mathrm{~mm}^{3}$ are risk factors for failure of laparoscopic cholecystectomies. In addition, factors related to the surgeon must be taken into account.
\end{abstract}

\section{Key Words}

Cholecystectomy, laparoscopic cholecystectomy, leukocytes, conversion to open surgery, complications 


\section{INTRODUCCIÓN}

La colecistitis aguda es una inflamación de la pared vesicular y constituye la segunda causa de abdomen agudo en el mundo y uno de los principales motivos de consulta en las salas de urgencia, donde cerca del $95 \%$ de los casos es por causa litiásica (1). El tratamiento para esta patología es netamente quirúrgico (2), y la colecistectomía laparoscópica (CL) es el procedimiento de elección (3). Esta puede sufrir conversión intraoperatoria debida a las complicaciones propias de la intervención, los factores del paciente o los asociados con el cirujano (4).

En Latinoamérica, la frecuencia de la conversión de la CL a colecistectomía abierta varía del $10 \%$ al $20 \%$ (5). El porcentaje de conversión a cirugía abierta en Estados Unidos es del 4,6\% en cirugía electiva y del 9,4\% en la de urgencia (6). En Colombia, algunos estudios indican que la conversión se presenta entre el $0,8 \%$ y el $12 \%$ de los casos $(7,8)$.

Existen múltiples factores predictores de conversión, tales como la edad, las patologías propias del paciente, las cirugías previas y el valor de leucocitos en el cuadro hemático (8). En un estudio realizado en el Hospital Universitario San Ignacio de Bogotá, encontraron que en cuanto a los factores de riesgo para la conversión de CL están el recuento de leucocitos (mayor de $10000 \mathrm{~mm}^{3}$ ) y la edad (mayor de 50 años) (7)

Otros estudios han determinado que la proteína C-reactiva (PCR) sirve como factor de riesgo predictor de conversión (9-11). La imagen ecográfica pueden ser de vital importancia a la hora de determinar la dificultad de la cirugía $(12,13)$ y el entrenamiento del personal de salud puede aumentar la probabilidad de alteración intraoperatoria (14). Las principales complicaciones que pueden presentarse en una cirugía convertida son: infección de la herida quirúrgica, hernias postoperatorias y adherencias (6).

Realizamos el presente estudio con el objetivo de establecer la frecuencia y los factores de riesgo asociados con las conversiones de CL a colecistectomía abierta, basándonos en los exámenes de laboratorio hematológicos y en la ecografía abdominal, así como en los factores sociodemográficos reportados en la historia de cada uno de los pacientes.

\section{MATERIALES Y MÉTODOS}

Se llevó a cabo un estudio descriptivo, de corte transversal, observacional y retrospectivo, durante el período comprendido entre el 1 de enero y el 3 de noviembre de 2015. Se revisó un total de 71 historias clínicas con diagnóstico de patología vesicular benigna, de pacientes sometidos a procedimiento quirúrgico vesicular en el Hospital Regional de Sogamoso, con el fin de describir, comparar y analizar distintos los factores que intervienen en el desenlace de conversión de la CL a la abierta. Se usó un muestreo no probabilístico por conveniencia. Los criterios de inclusión que se aplicaron fueron paciente sometido a colecistectomía, con resultados de laboratorio, incluidos reactantes de fase aguda como los leucocitos, resultados imagenológicos registrados en la historia clínica y diagnóstico de patología biliar benigna.

Los criterios de exclusión que se tuvieron en cuenta fueron datos incompletos y cirugía abdominal previa en otros hospitales.

Luego de identificadas y seleccionadas las variables de la investigación (sexo, edad, diagnóstico preoperatorio, exámenes de laboratorio [PCR, bilirrubinas totales y diferenciales, fosfatasa alcalina] y complicación intraoperatoria), se procedió a la recolección de datos de las historias clínicas y a la creación de una base de datos (programa de Excel 2007).

\section{Análisis estadístico}

Se presentaron los datos en tablas que contienen frecuencias absolutas y relativas (porcentajes). Se hace un análisis estadístico descriptivo univariado y de riesgo relativo bivariado para determinar el factor de riesgo asociado con la conversión de CL a cirugía abierta. Se usaron los programas de Excel 2007 (para la creación de una base de datos) y SPSS stadistics 22, usado para determinar los riesgos relativos.

\section{Consideraciones éticas}

De acuerdo con la resolución 8430 de 1993, mediante la cual se dictan los criterios de investigación en salud, se establece que esta es una investigación sin riesgo. Además, el Comité Ético de Investigación del Hospital, luego de evaluar y analizar el estudio, decidió aprobarnos el consentimiento informado para la revisión de historias clínicas y la ulterior publicación de los resultados.

\section{RESULTADOS}

Del total de 71 historias clínicas revisadas se excluyeron 12, dado que no cumplían con los criterios de inclusión. El manejo de todos los pacientes fue quirúrgico. En el 35,5\% de los pacientes se realizó una CL, en la que fue necesario convertir a cirugía convencional al $42,8 \%$ de los pacientes. Las causas por las que se requirió la conversión, según lo referido en el informe quirúrgico, fueron: adherencias epiploicas $(66,6 \%)$; no visualización del cístico $(11,1 \%)$; plastrón vesicular (11,1\%); fístula del cístico $(11,1 \%)$.

La mayoría de pacientes intervenidos fueron mujeres $(72,8 \%)$, pero la conversión a colecistectomía abierta predominó en el sexo masculino, con un $100 \%$, a diferencia del $36,8 \%$ que fue convertido en el sexo femenino. 
La edad promedio fue de 45,6 años (tabla 1) y los diagnósticos preoperatorios encontrados fueron colelitiasis $(98,3 \%)$, colecistitis aguda $(38,9 \%)$, coledocolitiasis $(6,7 \%)$ y otros $(37,8 \%)$. Teniendo en cuenta que un paciente podía tener múltiples diagnósticos de patología de vesícula biliar benigna, se encontró que el $32,2 \%$ presentó una patología y el $67,8 \%$ restante presentó patologías concomitantes, de los cuales, casi la mitad (45,7\%) exhibieron dos patologías.

Tabla 1. Edad promedio según sexo

\begin{tabular}{ll}
\hline$x$ edad hombres & 50,125 \\
x edad mujeres & 43,907 \\
\hline
\end{tabular}

De las patologías anteriormente mencionadas, el mayor porcentaje de conversión se encontró en los pacientes con colelitiasis aguda (100\%), seguido por el de la colecistitis aguda, con un $44,4 \%$. El diagnóstico de colelitiasis se hizo a través de ecografía abdominal en el $100 \%$ de los pacientes estudiados. Además, mediante este método se obtuvieron las medidas de los cálculos biliares, siendo el menor de $1 \mathrm{~mm}$ de diámetro y el mayor de $42 \mathrm{~mm}$. No hubo una relación significativa entre el tamaño de los cálculos y la dificultad quirúrgica, puesto que el tamaño promedio del cálculo fue de $15,5 \mathrm{~mm}$ en los convertidos y de 15,6 mm en los no convertidos. El diagnóstico de colecistitis aguda se hizo mediante ecografía abdominal en un $26,09 \%$. Con el uso de los criterios de Tokio, aumentó la sensibilidad al $85 \%$ y mediante esta se determinó que el $71 \%$ era colecistitis grado II (aguda moderada), lo que indicó que su carácter inflamatorio es importante y dificulta la tarea del cirujano.

El análisis estadístico de riesgo relativo bivariado nos arrojó importantes resultados. En cuanto a estos cabe destacar que los pacientes con edad $>50$ años tienen un $55 \%$ más de riesgo de ser convertidos de CL a cirugía abierta (odds ratio $[\mathrm{OR}]=0,55)$, siendo este resultado estadísticamente significativo $(\mathrm{p}<0,05)$. Además, según los resultados de laboratorio, la mayoría $(88,9 \%)$ de los pacientes convertidos tenía leucocitosis (niveles de leucocitos mayores de 10 $000 \mathrm{~mm}^{3}$ ), lo que indica que los pacientes con leucocitosis tienen un $40 \%$ más de riesgo de ser convertidos $(\mathrm{OR}=$ $0,40)$, siendo estadísticamente significativo $(\mathrm{p}<0,05)$.

\section{DISCUSIÓN}

Al igual que el estudio realizado en el Hospital Universitario San Ignacio de Bogotá, donde encontraron que la leucocitosis y la edad avanzada son factores que determinaron la conversión de CL a colecistectomía abierta, en el presente estudio se demostró que estos dos factores son determinan- tes en la conversión quirúrgica que requieren los pacientes $y$, por ende, en el tiempo de estancia hospitalaria (6).

Epidemiológicamente se encontró que las patologías vesiculares benignas son más frecuentes en el género femenino, esto debido a distintos factores hormonales, los cuales están probablemente relacionados con la influencia de los estrógenos (15), que constituye una de las causas por las que se realizan más colecistectomías a mujeres que a hombres. A partir de los resultados, se hizo evidente que la necesidad de conversión quirúrgica es mayor en el sexo masculino. Esto tiene justificación en que en los hombres, las adherencias epiploicas y la no visualización del cístico fueron más frecuentes que en las mujeres (16). Además, el tejido adiposo subcutáneo es más abundante en el sexo masculino, lo que dificultad aún más la tarea del cirujano y, en algunas ocasiones, lo obliga a cambiar la intervención por una colecistectomía abierta (17).

Según la literatura, los parámetros ecográficos preoperatorios (grosor de la pared vesicular, número y tamaño de los cálculos biliares) son los mejores indicadores de dificultad operatoria para la colecistectomía laparoscópica (13). El diagnóstico de colelitiasis mediante imagen ecográfica es bastante efectivo (18), mientras que el diagnóstico de colecistitis aguda tiene criterios ecográficos establecidos, como: grosor de la pared vesicular igual o mayor de $4 \mathrm{~mm}$, presencia de edema de la pared vesicular, litiasis intravesicular proyectada en el cuello, tamaño superior a 100 × $60 \mathrm{~mm}$, líquido perivesicular, colédoco dilatado y bilis de estasis, lo que demuestra que es muy dependiente del operador $(13,18,19)$. Sin embargo, cabe resaltar que en la investigación realizada no se encontró relación de la imagen ecográfica con la conversión de CL por falta de datos en los reportes imagenológicos.

Otros estudios han determinado que la PCR sirve como factor predictor de conversión $(17,20,21)$, pero no fue posible determinar en este estudio la asociación de más resultados de laboratorio como la PCR, por inexistencia al momento de realizar la búsqueda en la historia clínica, puesto que el médico no lo solicita en la mayoría de las ocasiones por ausencia de un protocolo en la institución.

Se establece, entonces, que estos factores son determinantes en el momento de decidir qué tipo de intervención quirúrgica requiere un paciente. Con el análisis previo de estos factores se puede evitar que un paciente tenga más de una intervención quirúrgica (10), lo que disminuye las posibles complicaciones que pueden presentarse por la conversión, como la infección de la herida quirúrgica, las hernias postoperatorias y las adherencias (22), y de esta manera evitar también una estancia hospitalaria más prolongada y un aumento de los costos de la hospitalización (9).

La principal limitación que tuvimos en el estudio fue la muestra poblacional pequeña, la cual se podría reproducir ampliando el tamaño de muestra. 


\section{CONCLUSIONES}

La conversión a cirugía abierta no debe interpretarse nunca como un fracaso, sino como una decisión en beneficio para el paciente, a pesar de los riesgos que conlleva. Para evitar esto y mediante los resultados de nuestro estudio, podemos concluir que la conversión es mayor en el sexo masculino, la edad por encima de los 50 años incrementa el riesgo y, por último, todos los exámenes de laboratorio que demuestren proceso inflamatorio agudo son también determinantes, principalmente los valores de leucocitos elevados incrementan este riesgo de conversión quirúrgica.

\section{Agradecimientos}

Al Dr. Moreno, patólogo y jefe de Calidad Académica del Hospital regional de Sogamoso, por su colaboración en las correcciones sugeridas. También a los trabajadores de archivos, por la búsqueda de las historias clínicas.

\section{REFERENCIAS}

1. Beyrouti MI, Beyrouti R, Affes N, et al. The lithiasic acute cholecystitis: laparoscopic treatment (About 106 cases). Tunis Med. 2011;89(4):355-9.

2. Almora CL, Arteaga Y, Plaza T, et al. Diagnóstico clínico y epidemiológico de la litiasis vesicular. Revisión bibliográfica. Rev Ciencias Médicas. 2012;16(1):200-14.

3. IbáñezA, Escalona P, DevaudJ, et al. Colecistectomía laparoscópica: experiencia de 10 años en la Pontificia Universidad Católica de Chile. Rev Chil Cir. 2007;59(1):10-5.

4. Ocádiz J, Blanco J, García A, et al. Conversión de la colecistectomía laparoscópica, más allá de la curva de aprendizaje. Acta Med Grupo Ángeles. 2011;9(4):192-5.

5. Zuluaga L, Clavijo W, Tavera A. Colecistectomía laparoscópica ambulatoria en una unidad quirúrgica no hospitalaria. Rev Colomb Cir. 2000;15:2-7.

6. Márquez F, Peláez D, Pezzano E, et al. Comportamiento de factores de riesgo de conversión de la Colelap a colecistectomía abierta. Hospital General de Barranquilla, enero de 2014-abril de 2015. Biociencias. 2015;10(2):81-8.

7. Vergnaud J, Penagos S, Lopera C, et al. Colecistectomía laparoscópica: experiencia en hospital de segundo nivel. Rev Colomb Cir. 2000; 15:8-13.

8. Granados J, Nieva R, Olvera G, et al. Criterios de conversión de cirugía laparoscópica a cirugía abierta y complicaciones poscolecistectomía: Una estadificación preoperatoria. Asoc Mex Cir Endosc. 2001;2(3):134-41.
9. Díaz S, Correa M, Giraldo L, et al. Experiencia en colecistectomía por laparoscopia en la Clínica Universitaria CES. Rev Colomb Cir. 2012;27:275-80.

10. Haleem S, Ansari M, Mussarat J, et al. Comparative assessment of TNF-alpha and C-reactive protein in patients subjected to open instead of laparoscopic cholecystectomy. Indian J Surg. 2007;69(3):99-104.

11. Jessica Mok K, Goh Y, Howell L, et al. Is C-reactive protein the single most useful predictor of difficult laparoscopic cholecystectomy or its conversion? A pilot study. J Minim Access Surg. 2016;12(1):26-32.

12. Bejarano M. Exactitud diagnóstica de la ecografía en patología vesicular. Rev Cir Col. 2014;17(4):207-12.

13. Pinto Paz M. Relación entre la ecografía preoperatoria y la dificultad de la colecistectomía laparoscópica Hospital Militar Central. Rev Gastroenterol. 2002;22(2).

14. Torres CJR, Torres LE, Weber SA, et al. Entrenamiento y curva de aprendizaje en colecistectomía laparoscópica y abierta. Resultados de la Encuesta Nacional de Lesiones de la Vía Biliar. Cir Gen. 2007;29(2):100-8.

15. Naranjo Rodríguez A, Rodríguez Ramos C. Litiasis biliar, colangitis aguda y colecistitis aguda. En: Ponce J, Castells A, Gomollon F (editores). Tratamiento de las enfermedades gastroenterológicas. 3. a edición. Madrid: Elsevier España S.A.; 2011. p. 415-26.

16. Sakpal S, Bindra S, Chamberlain R. Laparoscopic cholecystectomy conversion rates two decades later. JSLS. 2010;14(4):476-83.

17. Pedrosa Mendoza LE, Vázquez Ríos BS. Imagenología. La Habana: Editorial Ciencias Médicas; 2005.

18. Pérez MJ, Cabrera W, Varela G, et al. Distribución regional de la grasa corporal. Uso de técnicas de imagen como herramienta de diagnóstico nutricional. Nutr Hosp. 2010;25(2):207-23.

19. Kanakala V, Borowski D, Pellen M, et al. Risk factors in laparoscopic cholecystectomy: a multivariate analysis. Int J Surg. 2011;9(4):318-23.

20. Lin C, Collins J, Britt R, et al. Initial cholecystectomy with cholangiography decreases length of stay compared to preoperative MRCP or ERCP in the management of choledocholithiasis. Am Surg. 2015;81(7):726-31.

21. Domínguez L, Rivera A, Bermúdez C, et al. Análisis de los factores de conversión durante colecistectomía laparoscópica a abierta en una cohorte prospectiva de 703 pacientes con colecistitis aguda. Cir Esp. 2011;89(5):300-6.

22. Straatman J, Harmsen A, Cuesta $M$, et al. Predictive value of C-reactive protein for major complications after major abdominal surgery: A systematic review and pooled-analysis. 2015;10(7):e0132995. 